\title{
Reversible 3D optical data storage and information encryption in photo-modulated transparent glass medium
}

\author{
Zhen $\mathrm{Hu}^{1}$, Xiongjian Huang ${ }^{2}$, Zhengwen Yang ${ }^{1 凶}$, Jianbei Qiu', Zhiguo Song ${ }^{1}$, Junying Zhang $\mathbb{B}^{3 凶}$ and \\ Guoping Dong $\mathbb{1 0}^{2 \times}$
}

\begin{abstract}
Transparent glass has been identified as a vital medium for three-dimensional (3D) optical information storage and multi-level encryption. However, it has remained a challenge for directly writing 3D patterning inside a transparent glass using semiconductor blue laser instead of high-cost femtosecond laser. Here, we demonstrate that rare earth ions doped transparent glass can be used as 3D optical information storage and data encryption medium based on their reversible transmittance and photoluminescence manipulation. The color of tungsten phosphate glass doped with rare earth ions change reversibly from light yellow to blue upon alternating $473 \mathrm{~nm}$ laser illumination and temperature stimulation, resulting in the reversible luminescence modulation. The information data could be repeatedly written and erased in arbitrary 3D space of transparent glass, not only showing the ability of the excellent reproducibility and storage capacity, but also opening opportunities in information security. The present work expands the application fields of luminescent glass, and it is conducive to develop a novel 3D data storage and information encryption media.
\end{abstract}

\section{Introduction}

The advent of the information age is accompanied by the rapid growth of digital information, which is in urgent need for the development of huge storage space and security media. Optical storage technology with the huge storage capacity and low cost has become a new choice for information storage ${ }^{1,2}$. At present, the commercial optical storage devices mainly include Blu-ray discs, digital versatile discs, and compact discs ${ }^{3-5}$. However, due to the optical diffraction limitation, the optical storage technology faces a storage capacity bottleneck. To improve storage density, the traditional approach uses reducing laser

\footnotetext{
Correspondence: Zhengwen Yang (yangzw@kust.edu.cn) or

Junying Zhang (zjy@buaa.edu.cn) or Guoping Dong (dgp@scut.edu.cn)

${ }^{1}$ College of Materials Science and Engineering, Kunming University of Science and Technology, 650093 Kunming, China

${ }^{2}$ State Key Laboratory of Luminescent Materials and Devices, School of

Materials Science and Engineering, South China University of Technology,

510640 Guangzhou, China
}

Full list of author information is available at the end of the article wavelength or increasing numerical aperture of objective lens ${ }^{6-9}$. Recently, the super-resolution optical technology has been developed to break through the limit of optical diffraction ${ }^{10-12}$. Meanwhile, the three-dimensional (3D) information storage medium has been a good selection for improving the information storage capacity and extending storage life ${ }^{13-17}$. At present, the transparent glass has been proved as a viable information recording 3D medium under the processing of near-infrared femtosecond $\operatorname{laser}^{14,15,18}$. However, the femtosecond laser is a complicated and expensive equipment in contrast to the visible continuous semiconductor laser, and it usually requires a higher photon energy and a more complex operating process. In addition, the photostimulated luminescence particles or metal nanoparticles embedded in glasses were explored as an optical storage medium ${ }^{19}$. For the photostimulated luminescence particles based on the design of defect traps, it is relative difficult for the preparation of the photostimulated luminescence particles embedded

\section{(c) The Author(s) 2021}

\footnotetext{
(c) Open Access This article is licensed under a Creative Commons Attribution 4.0 International License, which permits use, sharing, adaptation, distribution and reproduction cc) in any medium or format, as long as you give appropriate credit to the original author(s) and the source, provide a link to the Creative Commons license, and indicate if changes were made. The images or other third party material in this article are included in the article's Creative Commons license, unless indicated otherwise in a credit line to the material. If material is not included in the article's Creative Commons license and your intended use is not permitted by statutory regulation or exceeds the permitted use, you will need to obtain permission directly from the copyright holder. To view a copy of this license, visit http://creativecommons.org/licenses/by/4.0/.
} 


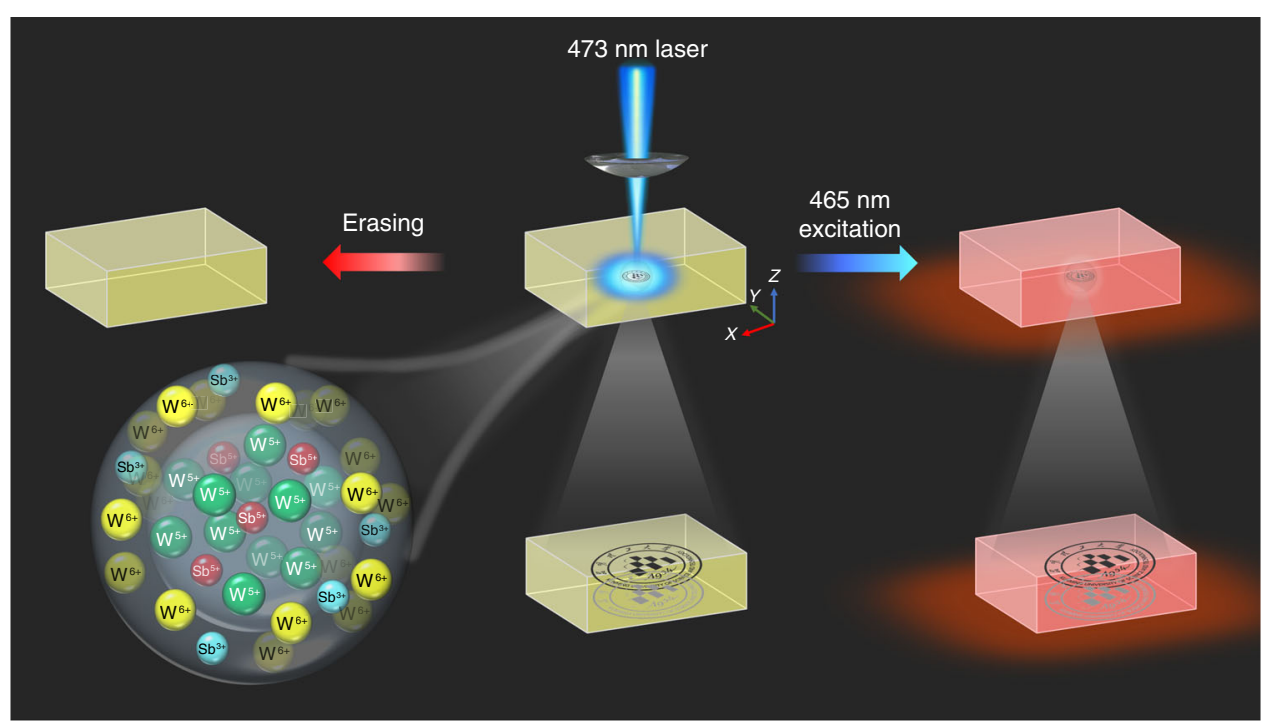

Fig. 1 Schematic of the writing, reading, and erasing of optical information. A focused $473 \mathrm{~nm}$ laser beam is irradiated into the glass, and the blue area is the photochromic region of the glass. The 3D optical data are written in transparent glass by a computer-controlled 3D XYZ translation stage. A $465 \mathrm{~nm}$ light from xenon lamp is used to excite the glass, and the photochromism-induced luminescence modification is recorded by a CCD camera. The transmittance or luminescence modulation is recovered by the thermal stimulation.

glasses $^{20}$. Thus these characteristics urge us to develop a new information recording technology for the bulk glass medium.

The application of rare earth doped materials can be extended based on their luminescence modification induced by the external field stimulation such as electric, magnetic and temperature fields ${ }^{21-24}$. Compared with the modification approaches of the photoluminescence of rare earth ions based on the external temperature, electric and magnetic fields, the external light field approach for photoluminescence modification of rare earth ions exhibits some advantages such as simple manipulation, real time, high efficiency, high safety and good repeatability. At present, the reversible photoluminescence modification of rare earth doped ceramics or films were demonstrated based on photochromic effect, exhibiting the potential application in some fields such as holographic memory and optical data storage ${ }^{25-29}$. However, 3D optical data storage cannot be achieved in the photocontrol ceramics due to their opacity. Compared to the photochromic ceramics, the photochromic bulk glasses are easier to realize the 3D optical data storage due to their transparent property, which improves the capacity of data storage. Therefore, the development of photomodulated glass is urgently needed to realize the $3 \mathrm{D}$ optical information storage applications.

The $\mathrm{WO}_{3}$ photochromic materials have been extensively exploited in amorphous films, powder, and polycrystalline ceramics ${ }^{30}$. The change of $\mathrm{W}^{6+}$ valence state to $\mathrm{W}^{5+}$ is responsible for the photochromism of $\mathrm{WO}_{3}$ materials ${ }^{17,23}$. In addition, the previous results have demonstrated that the phosphate glass is one of the most prospective photoluminescent matrixes for rare earth ions, which is mainly due to its high rare earth ion solubility, low nonlinear refractive index, large emission crosssection, etc ${ }^{31}$. In this study, the multifunctional tungsten phosphate glasses doped with rare earth ions were designed and prepared. The color of rare earth ions doped glass reversibly changes from light yellow to blue by alternating $473 \mathrm{~nm}$ laser illumination and thermal stimulation due to the change of $\mathrm{W}^{6+}$ valence state (Fig. 1), showing the writing and erasing ability. The reading out of optical information can be achieved by the transmittance and photoluminescence modulation of the rare earth doped glasses (Fig. 1). It is interesting that the optical information can be recorded in arbitrary 3D space of transparent glass, and the optical information written in the 3D space of transparent glass can be hierarchically discriminated, and thus the encryption function can be obtained.

\section{Results and discussion}

Laser irradiation may induce defects in glass, which could bring photoluminescence ${ }^{32,33}$. No luminescence was observed in the pure tungsten phosphate glass before and after $473 \mathrm{~nm}$ laser irradiation (Fig. S1, Fig. S2), indicating the absence of intrinsic luminescence centers or irradiation-induced luminescence defects. The photoluminescence intensity of the $\mathrm{Eu}^{3+}$ doped tungsten phosphate glass showing a typical $\mathrm{Eu}^{3+}$ ions emission pattern is dependent on the concentration of $\mathrm{Eu}^{3+}$. Doping concentration of $\mathrm{Eu}^{3+}$ is determined to be $1 \mathrm{~mol} \%$ 
in the tungsten phosphate glass (Fig. S1). The $\mathrm{Sb}_{2} \mathrm{O}_{3}$ addition has a significant influence on the transparency of the tungsten phosphate glasses. The glass without $\mathrm{Sb}_{2} \mathrm{O}_{3}$ is blue with low transparency. After adding $\mathrm{Sb}_{2} \mathrm{O}_{3}$, the glass exhibits high transparency in the region from $450 \mathrm{~nm}$ to $1800 \mathrm{~nm}$ (Fig. S3a). The glass with $1 \mathrm{~mol} \%$ $\mathrm{Sb}_{2} \mathrm{O}_{3}$ exhibits the best transparency and strongest red luminescence (Fig. S3a, b). The poor transparency of pure glass without $\mathrm{Sb}_{2} \mathrm{O}_{3}$ is attributed to the hopping of polarons from $\mathrm{W}^{6+}$ to $\mathrm{W}^{5+34,35}$. The $\mathrm{Sb}_{2} \mathrm{O}_{3}$ addition stabilizes the valence state of $\mathrm{W}^{6+}$, preventing the transformation from $\mathrm{W}^{6+}$ to $\mathrm{W}^{5+}$ (Fig. S3c, d). Thus the transparent tungsten phosphate glasses are obtained. The glass with the molar composition of $50 \mathrm{WO}_{3}$ $39.5 \mathrm{NaH}_{2} \mathrm{PO}_{4}-8 \mathrm{BaF}_{2}-0.5 \mathrm{Na}_{2} \mathrm{CO}_{3}-1 \mathrm{Sb}_{2} \mathrm{O}_{3}-1 \mathrm{EuF}_{3}$ was designed and prepared, which is denoted as WPG-Sb1.

The coloration of WPG-Sb1 glass was carried out under the scanning of the $473 \mathrm{~nm}$ laser, and the moving platform of the optical microscope was used to control the laser irradiation time on each point of the glass surface (Fig. 1). Figure 2a presents the transmission spectra and photos of WPG-Sb1 glass irradiated by the unfocused $473 \mathrm{~nm}$ laser $\left(151.26 \mathrm{~W} \mathrm{~cm}^{-2}\right)$ with a spot size of $1 \mathrm{~mm}$ for the various durations. The transmittance of the regions from 500 to $1400 \mathrm{~nm}$ of the glass starts to decrease when the laser irradiation time is about $1 \mathrm{~min}$. With the increase of the irradiation time, the transmittance of the glass further decreases, and the blue color becomes deeper until achieving saturation after $10 \mathrm{~min}$. The influence of $473 \mathrm{~nm}$ laser power density (irradiation time is $15 \mathrm{~min}$ ) on the transmission spectra and photos of WPG-Sb1 glass is shown in Fig. S4a. At the same irradiation time, the transmittance decreases with the increase of the laser power density.

The photochromism reaction of WPG-Sb1 glass can be used to modulate its luminescence property. Figure $2 \mathrm{~b}$ exhibits the luminescence spectra of WPG-Sb1 before and after the $473 \mathrm{~nm}$ laser $\left(151.26 \mathrm{~W} \mathrm{~cm}^{-2}\right)$ irradiation for various durations under the $465 \mathrm{~nm}$ excitation. As expected, the luminescence intensity of photochromic WPG-Sb1 glass gradually decreases with the irradiation time increase of $473 \mathrm{~nm}$ laser, and reaches stability at the irradiation time of $15 \mathrm{~min}$. The luminescence modulation degree $\left(\Delta R_{\mathrm{m}}\right)$ of WPG-Sb1 glass is expressed by the $\Delta R_{\mathrm{m}}$ $=\left(R_{0}-R_{\mathrm{i}}\right) / R_{0} \times 100 \%$, where the $R_{0}$ and $R_{\mathrm{i}}$ is the $614 \mathrm{~nm}$ initial luminescence intensity of raw WPG-Sb1 glass and the luminescence intensity of photochromic WPG-Sb1 glass treated by the $473 \mathrm{~nm}$ laser irradiation for various durations. The luminescence modulation degree increases with the increasing irradiation time of $473 \mathrm{~nm}$ laser (Fig. 2c). When the $473 \mathrm{~nm}$ irradiation times are 1, 3, 5, 7, 10 , and $15 \mathrm{~min}$, the calculated modulation degree of luminescence is $18 \%, 29 \%, 45 \%, 54 \%, 67 \%$ and $68 \%$, respectively. At the optimized $15 \mathrm{~min}$ irradiation time, the influence of $473 \mathrm{~nm}$ laser power density on the luminescence modification is demonstrated in Fig. S4b. The increase of $473 \mathrm{~nm}$ laser power density results in the modulation degree $\left(\Delta R_{\mathrm{m}}\right)$ increase (Fig. S4c).

The bleaching of photochromic WPG-Sb1 glass irradiated by the $473 \mathrm{~nm}$ laser at a power density of $151.26 \mathrm{~W}$ $\mathrm{cm}^{-2}$ for $15 \mathrm{~min}$ was explored by the heat stimulation at various temperatures for different durations. Figure $2 \mathrm{~d}$ exhibits the transmission spectra and photos of blue photochromic WPG-Sb1 glass heat-treated at various temperatures for $10 \mathrm{~min}$. When the heat-treatment temperature is $200^{\circ} \mathrm{C}$, the blue color of the glass starts to fade. At $300^{\circ} \mathrm{C}$, the blue color has been completely faded. The photochromism-induced luminescence quenching could gradually be recovered when the blue WPG-Sb1 glass was heat-treated at various temperatures for $10 \mathrm{~min}$ (Fig. 2e). The recovery degree $\left(\Delta R_{\mathrm{r}}\right)$ of $614 \mathrm{~nm}$ luminescence is characterized by the $\Delta R_{\mathrm{r}}=R_{\mathrm{t}} / R_{0}{ }^{*} 100 \%$, where the $R_{\mathrm{t}}$ and $R_{0}$ is the $614 \mathrm{~nm}$ luminescence intensity of the WPG-Sb1 photochromic glass heat-treated at various temperatures for $10 \mathrm{~min}$ and the $614 \mathrm{~nm}$ initial luminescence intensity of the raw WPG-Sb1 glass, respectively. The luminescence recovery degree increases with increasing the stimulation temperature (Fig. 2f). When the thermal stimulation temperature was $300^{\circ} \mathrm{C}$, the luminescence intensity of the glass was almost recovered to the corresponding original intensity.

The influence of thermal stimulation time at $200^{\circ} \mathrm{C}$ on the decoloration and luminescence recovery of blue glass was investigated (Fig. S5). The transmittance and luminescence of photochromic WPG-Sb1 glass increase with the increase of stimulation time. When the heattreatment time is $2 \mathrm{~h}$, the color and luminescence of photochromic WPG-Sb1 glass can be recovered to the initial state, respectively. The reversible photochromism of WPG-Sb1 glass was observed upon the $473 \mathrm{~nm}$ laser and thermal stimulation, respectively. In order to study the reproducibility, the WPG-Sb1 glass was treated by alternating irradiation of $473 \mathrm{~nm}$ laser $\left(151.26 \mathrm{~W} \mathrm{~cm}^{-2}\right.$, $15 \mathrm{~min})$ and thermal stimulus $\left(300^{\circ} \mathrm{C}, 10 \mathrm{~min}\right)$. As shown in Fig. 2g, the transmittance of the WPG-Sb1 glass can be switched off and on with excellent reproducibility after several cycles. The luminescence spectra of WPG-Sb1 upon alternating the $473 \mathrm{~nm}$ laser irradiation $(151.26 \mathrm{~W}$ $\left.\mathrm{cm}^{-2}, 15 \mathrm{~min}\right)$ and thermal stimulus $\left(300^{\circ} \mathrm{C}, 10 \mathrm{~min}\right)$ have been measured as a function of the cycle numbers (Fig. 2h), exhibiting no deterioration of the luminescence. 50 cycles of transmittance and luminescence of the WPGSb1 glass were carried out (Fig. S6). It can be seen that degradation to the quality of the signal was not observed, and the excellent reversible property has been obtained. This glass with double reversible regulation of transmittance and luminescence may have potential applications in the fields of data storage and information encryption. 

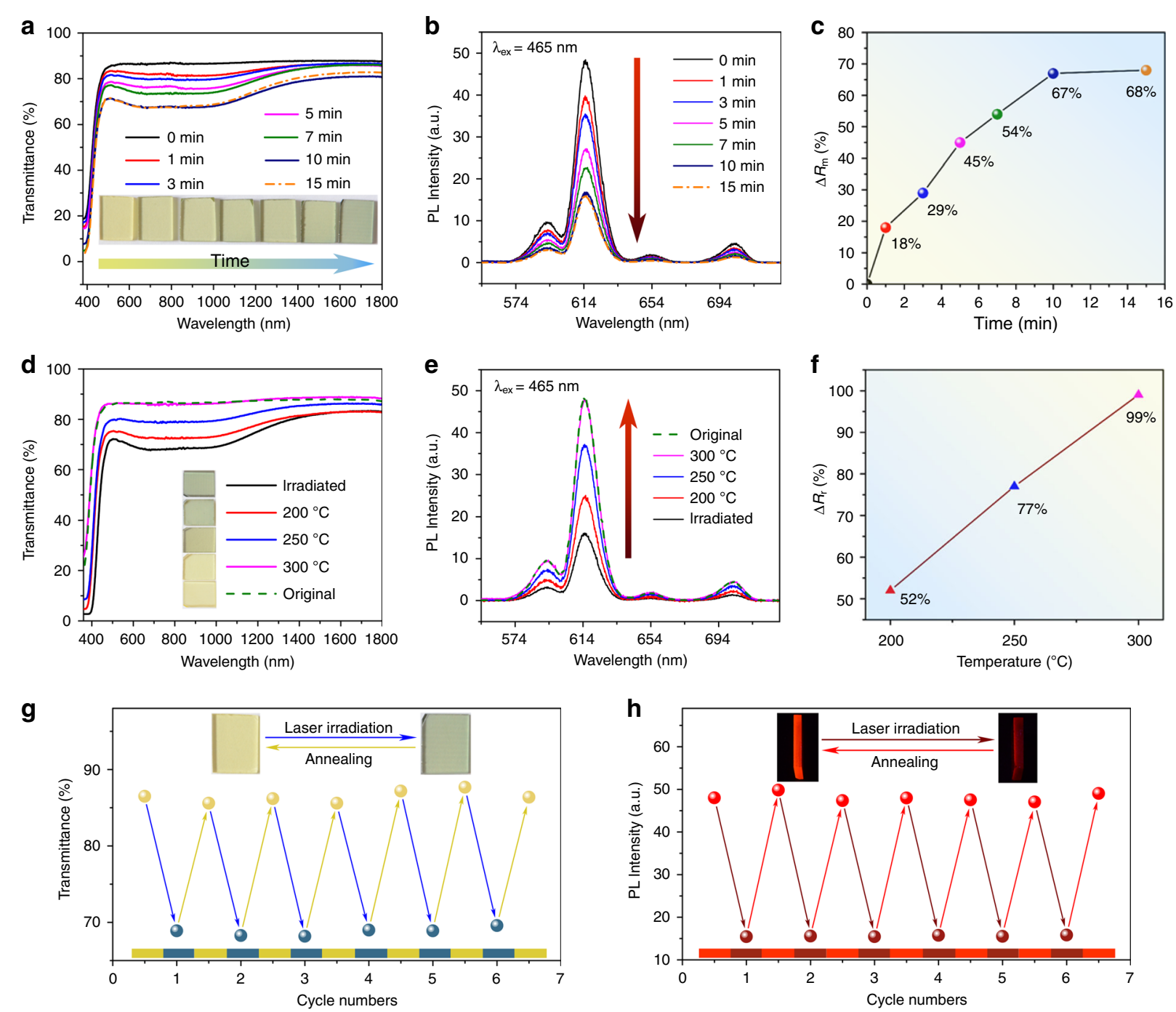

Fig. 2 Reversible photochromism and luminescence modification. a Transmission spectra and photos of WPG-Sb1 glass irradiated by unfocused $473 \mathrm{~nm}$ laser $\left(151.26 \mathrm{~W} \mathrm{~cm}^{-2}\right.$ ) for various times. b, c Luminescence spectra (b) and corresponding modulation degree (c) of WPG-Sb1 glass scanned by unfocused $473 \mathrm{~nm}$ laser for various times under $465 \mathrm{~nm}$ excitation. $\mathbf{d}$ Transmission spectra and photos of blue WPG-Sb1 glass heat-treated at various temperatures for $10 \mathrm{~min}$. e, $\mathbf{f}$ Luminescence spectra $(\mathbf{e})$ and luminescence recovery degree $\left(\triangle \mathrm{R}_{\mathrm{r}}\right)(\mathbf{f})$ of the blue WPG-Sb1 glass bleached by various temperatures for $10 \mathrm{~min}$. $\mathbf{g}, \mathbf{h} 614 \mathrm{~nm}$ transmittance (g) and luminescence intensity (h) of WPG-Sb1 glass by alternating $473 \mathrm{~nm}$ laser $\left(151.26 \mathrm{~W} \mathrm{~cm}^{-2}, 15 \mathrm{~min}\right)$ and thermal stimulation $\left(300^{\circ} \mathrm{C}, 10 \mathrm{~min}\right)$ as a function of cycle numbers.

As mentioned above, no luminescence from the glass host or defects are generated by the blue light irradiation in the tungsten phosphate glass. Therefore, the signal readout without interference can be obtained in the rare earth ions doped glass upon $465 \mathrm{~nm}$ excitation.

The reversible photochromism mechanism of the WPG-Sb1 glass was investigated by Raman, electron paramagnetic resonance (EPR), and X-ray photoelectron spectroscopy (XPS) spectra of the original, photochromic, or decolorated WPG-Sb1 glasses. The photochromism and decoloration of WPG-Sb1 glass were confirmed to be not attributed to the structural change of the glass (Fig. S7) or valence change of $\mathrm{Eu}^{3+}$ (Fig. S8). No peak of the oxygen vacancy was observed in the EPR spectra at room temperature of original, photochromic, and decolorated WPG-Sb1 glasses (Fig. S9), which suggests that the coloration of WPG-Sb1 glass is not attributed to the F-color center ${ }^{36,37}$. The grating structure and migration of elements were not observed in the photochromic region of WPG-Sb1 glass from the SEM image and energydispersive X-ray spectroscopy mapping (Fig. S10). The XPS spectra of W element in WPG-Sb1 before and after photochromism are shown in Fig. 3a, c. Two intensive 35.8 and $37.9 \mathrm{eV}$ peaks of $\mathrm{W}^{6+}$ and two weak 34.6 and $36.7 \mathrm{eV}$ peaks from $\mathrm{W}^{5+}$ were detected in the WPG-Sb1 glass before photochromism. It is interesting that the XPS 

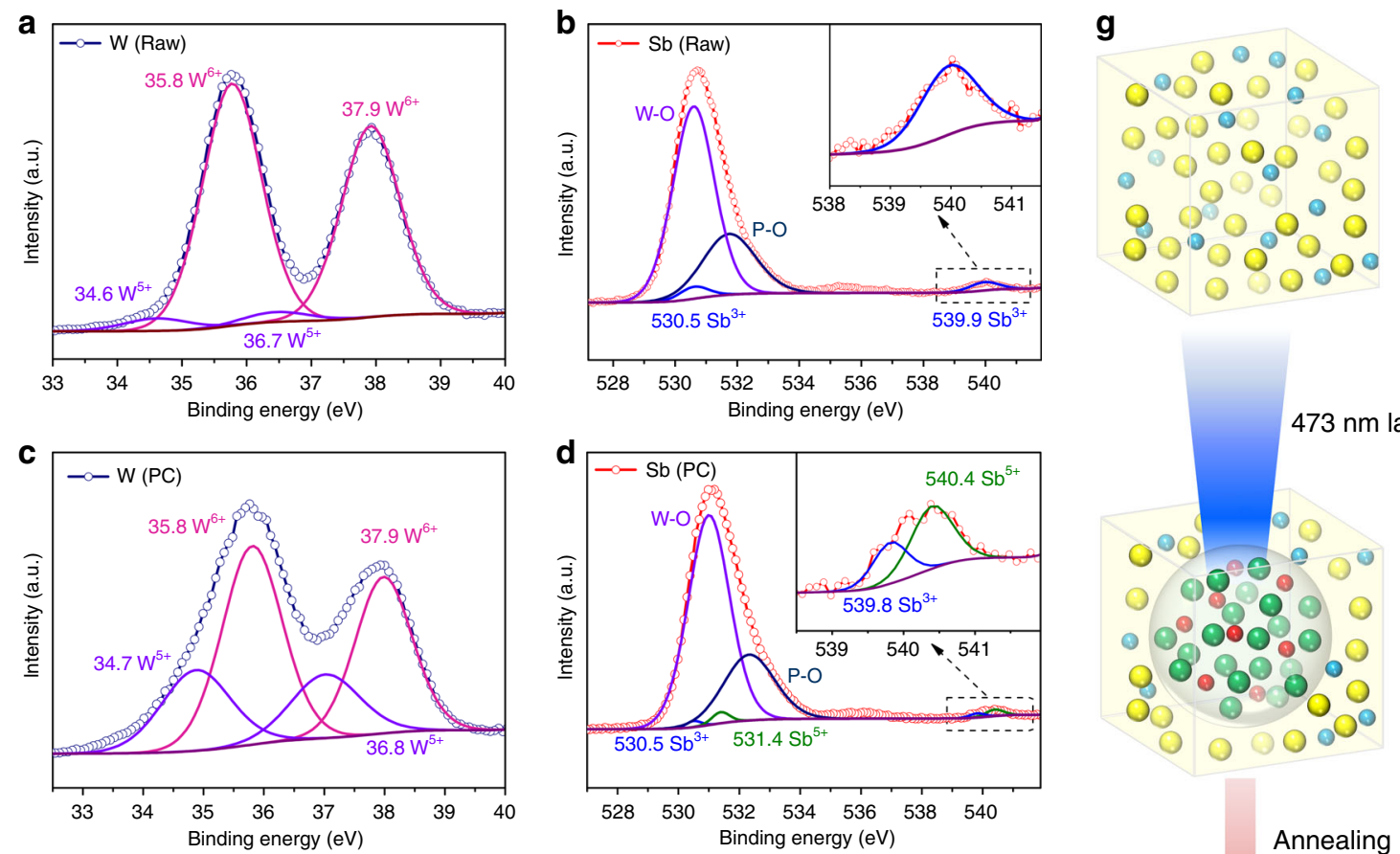

$473 \mathrm{~nm}$ laser
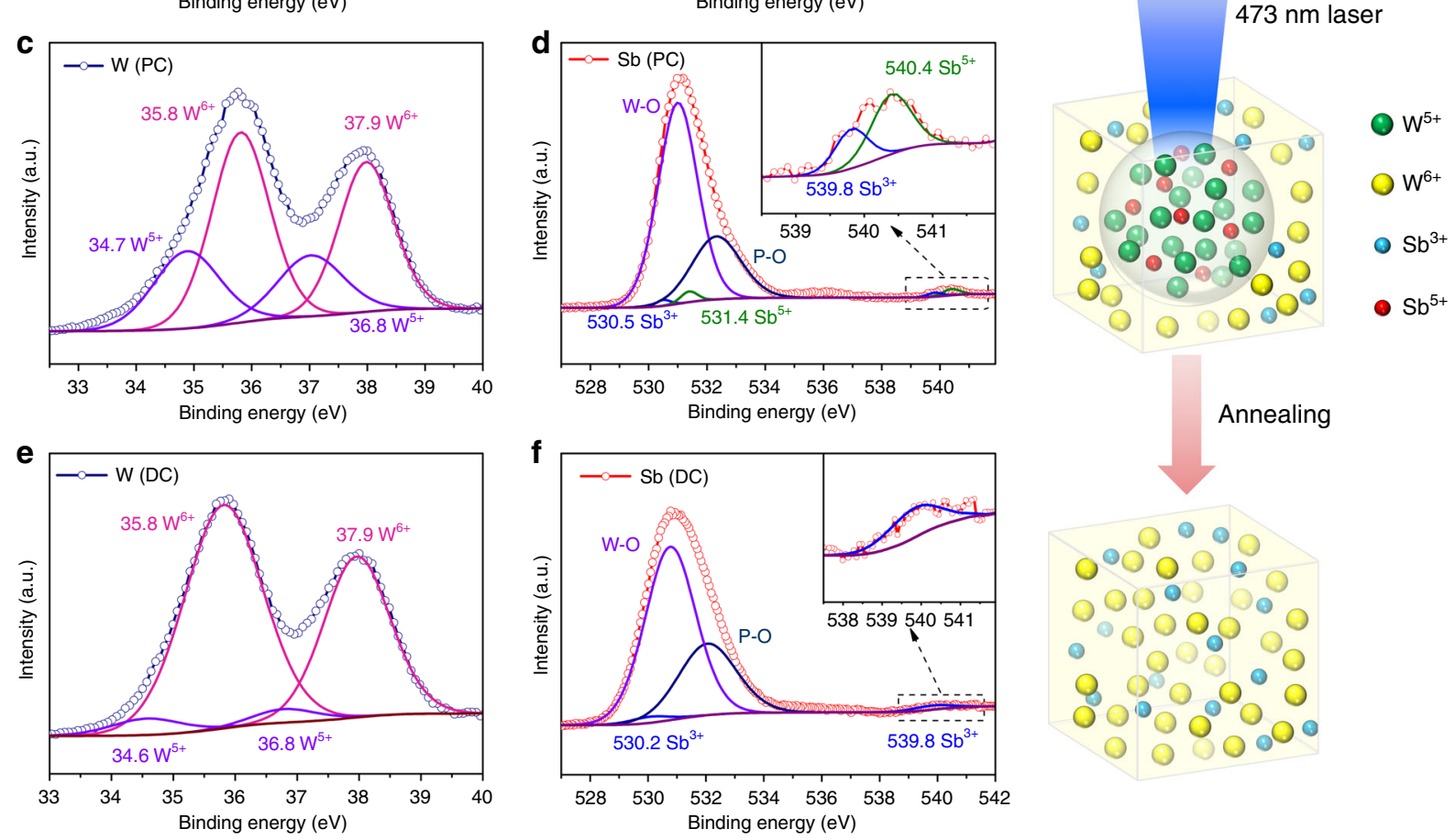

Annealing

Fig. 3 Reversible photochromic mechanism. a, c XPS spectra of W element in WPG-Sb1 before (a) and after (c) photochromism (PC) (151.26 W $\left.\mathrm{cm}^{-2}, 15 \mathrm{~min}\right)$. b, d XPS spectra of Sb element in WPG-Sb1 before (b) and after (d) photochromism $\left(151.26 \mathrm{~W} \mathrm{~cm}^{-2}, 15 \mathrm{~min}\right) . \mathbf{e}, \mathbf{f}$ XPS spectra of W (e) and $\mathrm{Sb}(\mathbf{f})$ elements in the decolorated (DC) glass caused by heat-treatment at $300^{\circ} \mathrm{C}$ for $10 \mathrm{~min}$. $\mathbf{g}$ Proposed reversible photochromism mechanism of tungsten phosphate glass.

peaks of $\mathrm{W}^{5+}$ significantly intensify in the photochromic WPG-Sb1 glass $\left(151.26 \mathrm{~W} \mathrm{~cm}^{-2}, 15 \mathrm{~min}\right)$. The results indicate that $473 \mathrm{~nm}$ laser irradiation induces the transformation from $\mathrm{W}^{6+}$ to $\mathrm{W}^{5+}$. XPS spectra of Sb element in WPG-Sb1 before and after photochromism are shown in Fig. 3b, d. 539.9 and $530.5 \mathrm{eV}$ binding energies of $\mathrm{Sb}^{3+}$ were detected in the XPS spectra, and no XPS peak of $\mathrm{Sb}^{5+}$ were observed in WPG-Sb1 before photochromism. While the typical XPS peaks of $\mathrm{Sb}^{5+}$ can be observed in the photochromic WPG-Sb1, which locate at 531.4 and $540.4 \mathrm{eV}^{38,39}$. The following reactions $\mathrm{Sb}^{3+}+h v \rightarrow \mathrm{Sb}^{5+}$ $+2 \mathrm{e}^{-}$and $\mathrm{W}^{6+}+h v+\mathrm{e}^{-} \rightarrow \mathrm{W}^{5+}$ may happen for the WPG-Sb1 glass upon $473 \mathrm{~nm}$ laser irradiation. The hopping of polarons from $\mathrm{W}^{6+}$ to $\mathrm{W}^{5+}$ is responsible for the photochromism of WPG-Sb1 glass due to the $\mathrm{W}^{5+}$ generation $^{34}$.
Other light sources such as $532\left(284.19 \mathrm{~W} \mathrm{~cm}^{-2}\right), 808$ $\left(78.53 \mathrm{~W} \mathrm{~cm}^{-2}\right)$, and $980 \mathrm{~nm}\left(53.47 \mathrm{~W} \mathrm{~cm}^{-2}\right)$ lasers were used to irradiate the WPG-Sb1 glass. We demonstrated that the WPG-Sb1 glass also exhibits the photochromic property upon $532 \mathrm{~nm}$ laser stimulation (Fig. S11), while no photochromism is observed upon 808 and $980 \mathrm{~nm}$ lasers stimulation. The photochromic effect of the WPGSb1 glass upon $473 \mathrm{~nm}$ laser irradiation is better than that by $532 \mathrm{~nm}$ laser irradiation at the same irradiation time (Fig. S11). $\mathrm{Sb}^{3+}$ exhibits a broad absorption ranging from $350 \mathrm{~nm}$ to $700 \mathrm{~nm}$ with a peak at $480 \mathrm{~nm}^{40}$. The reaction between $\mathrm{Sb}^{3+}$ and $\mathrm{W}^{6+}$ in the WPG-Sb1 glass may be related to the absorption of $\mathrm{Sb}^{3+}$ upon 473 or 532 laser irradiation. The WPG-Sb1 glass irradiated for $1 \mathrm{~h}$ by $465 \mathrm{~nm}$ light of xenon lamp exhibits no photochromism due to lower power density (Fig. S11). XPS spectra of 


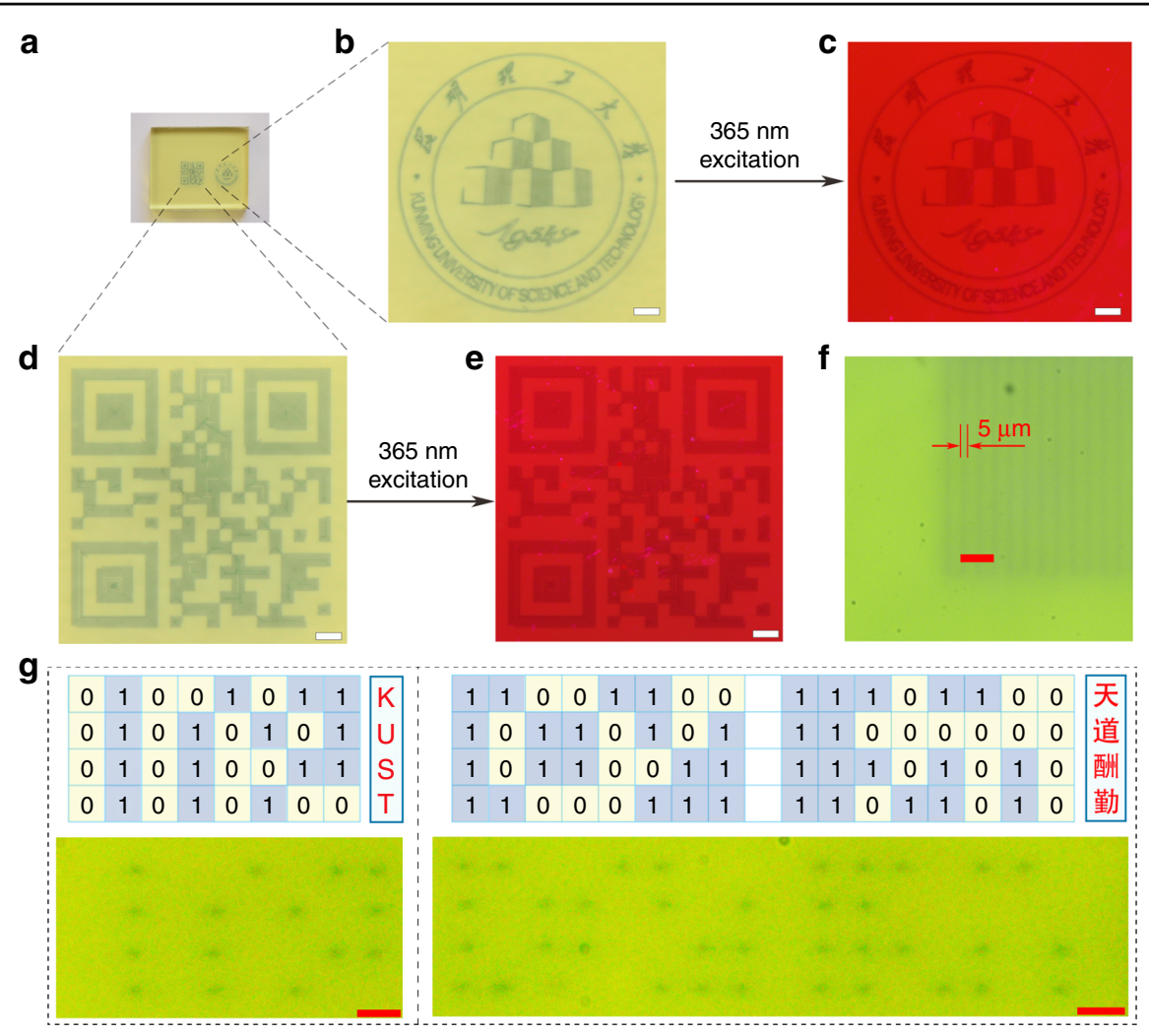

Fig. 4 Optical information writing and readout. $\mathbf{a}, \mathbf{b}$, $\mathbf{d}$ The raw $(\mathbf{a})$ and enlarged $(\mathbf{b}, \mathbf{d})$ photochromic logo patterns. $\mathbf{c}$, e The enlarged luminescent logo pattern upon the $365 \mathrm{~nm}$ excitation. $\mathbf{d}$, e The information data were hidden into the photochromic (d) or luminescent (e) two-dimensional code pattern, and the information "I love KUST" can be obtained by scanning the two-dimensional code pattern. Scale bar: $500 \mu \mathrm{m}$. f The scanning line width of focused 473 lasers. Scale bar: $40 \mu \mathrm{m}$. $\mathbf{g}$ The "KUST" alphabet (left) and the "tian dao chou qin" idiom (right) recorded into the transparent glass by binary format. Scale bar: $20 \mu \mathrm{m}$.

decolorated WPG-Sb1 glass after heat-treatment at $300^{\circ} \mathrm{C}$ for 10 min were measured. As shown in Fig. 3e, f, the XPS peak intensities of $\mathrm{W}^{6+}$ and $\mathrm{Sb}^{3+}$ in the decolorated WPG-Sb1 glass increase in comparison with those in the photochromism sample. By contrast, the XPS peak intensity of $\mathrm{W}^{5+}$ decreases in the decolorated WPG-Sb1 glass after heat-treatment, and the XPS peak of $\mathrm{Sb}^{5+}$ disappears. The $\mathrm{W}^{5+} \rightarrow \mathrm{W}^{6+}+\mathrm{e}^{-}$and $\mathrm{Sb}^{5+}+2 \mathrm{e}^{-} \rightarrow$ $\mathrm{Sb}^{3+}$ reactions take place in the photochromic WPG-Sb1 glass upon thermal stimulation. The thermal stimulation results in the decrease of $\mathrm{W}^{5+}$ amount in the photochromic WPG-Sb1 glass, causing its decoloration.

The luminescence of WPG-Sb1 has been successfully modulated by its photo-control transmittance. The mechanisms of radiative energy transfer and resonance energy transfer can be used to explain this luminescence modulation ${ }^{41,42}$. The radiative energy transfer and resonance energy transfer can be characterized by the decay lifetime of active centers. Figure S12 presents the decay curves of $614 \mathrm{~nm}$ emission of the glass upon $473 \mathrm{~nm}$ irradiation for different durations. The decay lifetime of $614 \mathrm{~nm}$ emission from $\mathrm{Eu}^{3+}$ slightly decreases with the increase of the irradiation time, which suggests that the luminescence modulation is not mainly attributed to the resonance energy transfer. The luminescence mechanism is presented in Fig. S13a. The radiative transitions from ${ }^{5} \mathrm{D}_{0}$ to ${ }^{5} \mathrm{~F}_{\mathrm{n}}(n=1,2,3$, and 4$)$ of $\mathrm{Eu}^{3+}$ produces the 593, 614, 650, and $700 \mathrm{~nm}$ luminescence, respectively. The luminescence of $\mathrm{Eu}^{3+}$ overlaps with the absorbance of the photochromic WPG-Sb1 glass (Fig. S13b). Thus the luminescence of $\mathrm{Eu}^{3+}$ can be absorbed by the blue glass host, resulting in the luminescence modulation.

In order to demonstrate the optical storage application of tungsten phosphate glasses, the as-prepared WPG-Sb1 glass was irradiated by the focused $473 \mathrm{~nm}$ laser. The used power density of focused $473 \mathrm{~nm}$ laser is about $1915 \mathrm{~kW}$ $\mathrm{cm}^{-2}$, below the $2058 \mathrm{~kW} \mathrm{~cm}{ }^{-2}$ power density threshold of glass damage (Fig. S14). The single scanning line width and spot size of focused 473 lasers are about $5 \mu \mathrm{m}$ (Fig. 4f, $\mathrm{g}$ ), and the resolution can be improved by the laser spot reduction. The laser irradiation time was shortened to $0.02 \mathrm{~s}$ to get the saturated photochromic WPG-Sb1 glass because the $473 \mathrm{~nm}$ laser focusing caused power density improvement. Photochromic complicated logo pattern 
was written at the surface of WPG-Sb1 glass by the focused laser direct writing technology (Fig. 4a, b). Based on the luminescence modification induced by the photochromism reaction, the luminescence logo pattern at the surface of WPG-Sb1 glass could also be revealed by using $365 \mathrm{~nm}$ UV lamp (Fig. 4c). It is interesting that the information data can be stored in the photochromic or luminescent two-dimensional code pattern (Fig. 4d, e). The reading out of information could be achieved by using intelligent mobile phone to scan the two-dimensional code pattern, as exhibited by the information "I love KUST" (Supplementary Video 1). The photochromic pattern can be obviously observed after ten months (Fig. S15), which suggests the photochromic glass has excellent chemical stability. Reading out of information can be obtained by the change of transmittance or luminescent intensity, which corresponds to " 0 " and "1" state in the binary system, respectively (Fig. 2g). The binary dot arrays encoded by common computer file formats (Fig. S16) were written into the transparent glass, for example to generate the binary data of "KUST" alphabet and the Chinese idiom of "tian dao chou qin" (Fig. 4g). The photochromic or luminescent pattern can be erased by the thermal stimulation, and the photochromic reversibility of the glass ensures its reusing (Fig. S17). In addition, other rare earth ions (such as $\mathrm{Dy}^{3+}$ ) doped glass with the composition of $50 \mathrm{WO}_{3}$ $39.5 \mathrm{NaH}_{2} \mathrm{PO}_{4}-8 \mathrm{BaF}_{2}-0.5 \mathrm{Na}_{2} \mathrm{CO}_{3}-1 \mathrm{Sb}_{2} \mathrm{O}_{3}-1 \mathrm{DyF}_{3}$ was prepared. The blue photochromism and yellow luminescence patterns of $\mathrm{Dy}^{3+}$ were obtained (Fig. S18), demonstrating the universality of the technology.

The inorganic photochromic glass exhibits many advantages such as the ability of 3D optical data storage due to its transparent property. As shown in Fig. 5a, the $3 \mathrm{D}$ optical information was written in various layers of the glass by the $473 \mathrm{~nm}$ laser direct writing technology, and the "KUST" four letters in the different layers can be observed by an optical microscope. The 3D optical data stored in the glass can be erased by the thermal stimulation (Fig. 5b). The "pentacle, quadrate, triangle, and circle" optical information was rewritten in the different layers of the glass (Fig. 5c), exhibiting the reversible 3D optical data storage ability of the glass. It is noted that the complicated 3D structure can be written inside the glass by the $473 \mathrm{~nm}$ direct laser writing technology (Fig. 5d), exhibiting the universality of the technology. In addition, the photo-modulated glass not only shows the ability of the reversible 3D optical data storage, but also opens a new opportunity in the information encryption. The "KUST" optical information written in various layers of the transparent glass can be hierarchically discriminated using an optical microscope (Supplementary Video 2), and thus the encryption function can be obtained.
In summary, the photochromism of rare earth ions doped tungsten phosphate transparent glass was demonstrated under the stimulation of $473 \mathrm{~nm}$ laser, which could be tailored by the laser power density or laser irradiation time. The decoloration of photochromic glass was obtained by thermal stimulation. The photochromism of rare earth ions doped tungsten phosphate transparent glass exhibited excellent reversibility, reproducibility and stability. The photoluminescence intensity was reversibly modified by the transmittance modulation of the rare earth ions doped glasses. The complex information patterns can be written and erased in the photo-modulated glass, showing the ability of the reversible 3D optical data storage. In addition, the optical information written in the arbitrary 3D space of transparent glass can be hierarchically discriminated, demonstrating the information encryption function. We believe that this photomodulated glass is of importance for extending its new applications in the optoelectronic fields.

\section{Materials and methods Glass preparation}

$\mathrm{WO}_{3}$ (99.99\%), $\quad \mathrm{BaF}_{2} \quad$ (99.99\%), $\quad \mathrm{Sb}_{2} \mathrm{O}_{3} \quad$ (99.99\%), $\mathrm{Na}_{2} \mathrm{CO}_{3}(99.99 \%), \mathrm{EuF}_{3}$ (99.99\%), $\mathrm{DyF}_{3}$ (99.99\%) and $\mathrm{NaH}_{2} \mathrm{PO}_{4}$ (99.0\%) were used as raw materials for the preparation of tungsten phosphate glasses doped with $\mathrm{Eu}^{3+}$ or $\mathrm{Dy}^{3+}$. Tungsten phosphate glasses with the molar compositions of $(51-x) \mathrm{WO}_{3}-39.5 \mathrm{NaH}_{2} \mathrm{PO}_{4}-8 \mathrm{BaF}_{2}$ $0.5 \mathrm{Na}_{2} \mathrm{CO}_{3}-x \mathrm{Sb}_{2} \mathrm{O}_{3}-1 \mathrm{EuF}_{3}(x=0,0.5,1$ and 2$)$ were prepared by the conventional melt-quenching method. The raw materials were mixed homogeneously in an agate mortar, and then were melted at $1050^{\circ} \mathrm{C}$ for $1 \mathrm{~h}$ in air. Then the melted tungsten phosphate glass was poured onto a brass mold and cooled down to room temperature. The obtained glass was annealed at $420^{\circ} \mathrm{C}$ for $5 \mathrm{~h}$ to remove thermal strains. Then polished glass with appropriate size was obtained before the laser irradiation. To investigate the influence of $\mathrm{Eu}^{3+}$ concentration on the luminescence, the $50 \mathrm{WO}_{3}-(40.5-y) \quad \mathrm{NaH}_{2} \mathrm{PO}_{4}-8 \mathrm{BaF}_{2}$ $0.5 \mathrm{Na}_{2} \mathrm{CO}_{3}-1 \mathrm{Sb}_{2} \mathrm{O}_{3}-y \mathrm{EuF}_{3} \quad(y=0,0.25,0.5,1.0,1.5$ and 2.0) tungsten phosphate glasses were prepared by the same approach.

\section{Reversible writing and erasing of optical information in the glass}

The coloration of glass was carried out under the irradiation of $473 \mathrm{~nm}$ laser with and without focusing. The $473 \mathrm{~nm}$ laser (MBL-N-473 nm-1 W) was focused by a $50 \times(\mathrm{NA}=0.55 ; \mathrm{WD}=8.2 \mathrm{~mm})$ objective lens (EPLE50). The resolution of the laser spot was about $5 \mu \mathrm{m}$. The focusing laser power density $\left(1915 \mathrm{~kW} \mathrm{~cm}^{-2}\right)$ is slightly below the threshold power density of glass damage. The optical shutter (SR470) was used to control the on/off of laser irradiation. The moving platform of the optical 

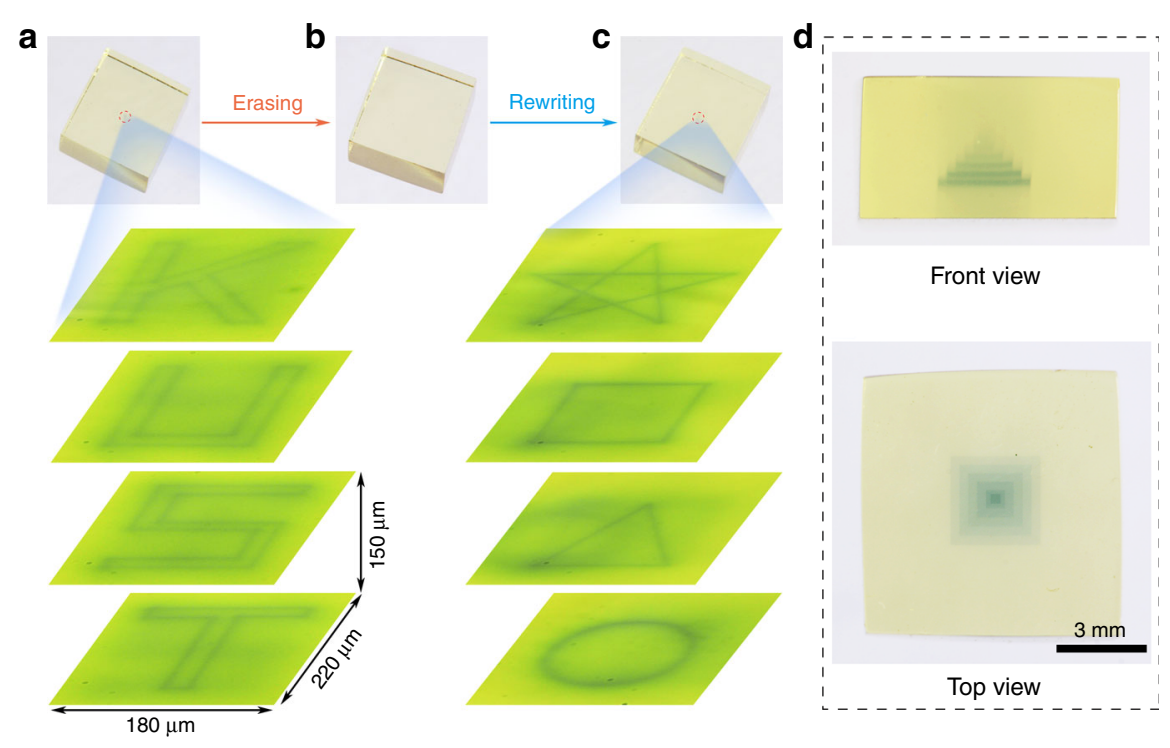

Fig. 5 Demonstration of 3D optical data storage and information encryption. a The 3D optical information written in various layers of the glass, the photograph of the transparent glass with the "KUST" information under the daylight (upper photo), and the optical microscope images of "KUST" information observed by using an optical microscope. $\mathbf{b}$ The photograph of glass after the "KUST" information erasing. $\mathbf{c}$ The 3D optical information rewritten in various layers of the glass, the photograph of the transparent glass with the "pentacle, quadrate, triangle and circle pattern" information under daylight (upper photo) and the optical microscope images of "pentacle, quadrate, triangle and circle" information observed by using optical microscope. $\mathbf{d}$ The 3D structure inside the glass from the front view and top view under daylight.

microscope (Axio Imager A1m) was used to control the laser irradiation time by using the computer software program to manipulate the movement speed of the glass. The optical data were erased by thermal stimulus at different temperatures and durations.

\section{Sample characterization}

The optical microscope images were captured by a Nikon (AZ100M) optical microscope equipped with a CCD camera. The photochromic images under daylight and photoluminescence images under the excitation of $365 \mathrm{~nm} \mathrm{UV}$ lamp were taken by a CCD camera (Nikon D7100, Japan). A mobile phone with a commercial APP official QR code Reader was used for QR code recognition (Supplementary video 1). The transmission spectra of tungsten phosphate glass samples before and after photochromism were measured by the U- 4100 spectrophotometer. The luminescence and excitation spectra of tungsten phosphate glass were measured by the HITACHIU-F-7000 spectrophotometer using Xe lamp as the light source. An Edinburgh FLS 980 instrument (Edinburgh Instruments Ltd., Livingston, UK) was used to measure the lifetime decay of the glasses. The chemical states of tungsten phosphate glass were char-

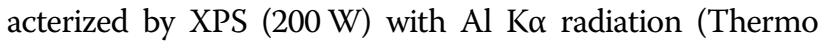
Fisher Scientific) under vacuum conditions. For the Raman spectra measurement of the samples, the Argon laser with continuous wave $(\lambda=514 \mathrm{~nm})$ was used as the excitation source. The Bruker model ELEXSYS-IIE500 spectrometer (Bruker, Switzerland) was used to measure the EPR spectra at room temperature. The element distribution was detected by a Zeiss sigma 300 scanning electron microscope with an energy-dispersive X-ray spectrometer (SmartEDX).

\section{Acknowledgements}

This work was supported by the National Natural Science Foundation of China $(51762029,62075063,51772101)$ and the Applied Basic Research Key Program of Yunnan Province (2018FA026), and the Key R\&D Program of Guangzhou (202007020003).

\section{Author details}

${ }^{1}$ College of Materials Science and Engineering, Kunming University of Science and Technology, 650093 Kunming, China. ${ }^{2}$ State Key Laboratory of Luminescent Materials and Devices, School of Materials Science and Engineering, South China University of Technology, 510640 Guangzhou, China. ${ }^{3}$ School of Physics, Beihang University, 100191 Beijing, China

\section{Author contributions}

Z.Y., J.Z. and G.D. conceived, designed, and supervised the overall project. Z.H. conducted the experiments with the help of X.H. Z.H. and Z.Y. wrote the manuscript. All the authors discussed the results and commented on the manuscript.

\section{Conflict of interest}

The authors declare no competing interests.

Supplementary information The online version contains supplementary material available at https://doi.org/10.1038/s41377-021-00581-y. 
Received: 18 February 2021 Revised: 27 May 2021 Accepted: 21 June 2021 Published online: 07 July 2021

\section{References}

1. Gu, M., Zhang, Q. M. \& Lamon, S. Nanomaterials for optical data storage. Nat. Rev. Mater. 1, 16070 (2016)

2. Kimel, A. V. \& Li, M. Writing magnetic memory with ultrashort light pulses. Nat. Rev. Mater. 4, 189-200 (2019).

3. Zhang, Q. M. et al. High-capacity optical long data memory based on enhanced Young's modulus in nanoplasmonic hybrid glass composites. Nat. Commun. 9, 1183 (2018).

4. Gu, M., Li, X. P. \& Cao, Y. Y. Optical storage arrays: a perspective for future big data storage. Light.: Sci. Appl. 3, e177 (2014).

5. Zhuang, Y. X. et al. Optical data storage and multicolor emission readout on flexible films using deep-trap persistent luminescence materials. Adv. Funct. Mater. 28, 1705769 (2018)

6. Scott, T. F. et al. Two-color single-photon photoinitiation and photoinhibition for subdiffraction photolithography. Science 324, 913-917 (2009).

7. Terris, B. D. et al. Near-field optical data storage using a solid immersion lens. Appl. Phys. Lett. 65, 388-390 (1994).

8. Gan, Z. S. et al. Three-dimensional deep sub-diffraction optical beam lithography with $9 \mathrm{~nm}$ feature size. Nat. Commun. 4, 2061 (2013).

9. Andrew, T. L., Tsai, H. Y. \& Menon, R. Confining light to deep subwavelength dimensions to enable optical nanopatterning. Science $\mathbf{3 2 4}$ 917-921 (2009).

10. Liu, Y. J. et al. Amplified stimulated emission in upconversion nanoparticles for super-resolution nanoscopy. Nature 543, 229-233 (2017).

11. Zhou, J. J. et al. Single-particle spectroscopy for functional nanomaterials. Nature 579, 41-50 (2020).

12. $L i, N$. J. et al. Achieving $N 20$ resolution by one-color initiation and deactivation of polymerization. Science 324, 910-913 (2009).

13. Parthenopoulos, D. A. \& Rentzepis, P. M. Three-dimensional optical storage memory. Science 245, 843-845 (1989).

14. Royon, A. et al. Silver clusters embedded in glass as a perennial high capacity optical recording medium. Adv. Mater. 22, 5282-5286 (2010).

15. Zhang, J. Y. et al. Seemingly unlimited lifetime data storage in nanostructured glass. Phys. Rev. Lett. 112, 033901 (2014).

16. Huang, X. J. et al. Reversible 3D laser printing of perovskite quantum dots inside a transparent medium. Nat. Photonics 14, 82-88 (2020).

17. Poirier, G. et al. Bulk photochromism in a tungstate-phosphate glass: a new optical memory material? J. Chem. Phys. 125, 161101 (2006).

18. Tan, D. Z. et al. Femtosecond laser induced phenomena in transparent solid materials: fundamentals and applications. Prog. Mater. Sci. 76, 154-228 (2016).

19. Qiu, J. R. et al. Manipulation of gold nanoparticles inside transparent materials. Angew. Chem. Int. Ed. 43, 2230-2234 (2004).

20. Lin, S. S. et al. High-security-level multi-dimensional optical storage medium: nanostructured glass embedded with $\mathrm{LiGa}_{5} \mathrm{O}_{8}: \mathrm{Mn}^{2+}$ with photostimulated luminescence. Light:: Sci. Appl. 9, 22 (2020).

21. Wong, M. C. et al. Temporal and remote tuning of piezophotonic-effectinduced luminescence and color gamut via modulating magnetic field. Adv. Mater. 29, 1701945 (2017).
22. Zhan, Y. H. et al. Electrochromism induced reversible upconversion luminescence modulation of $\mathrm{WO}_{3}: \mathrm{Yb}^{3+}, \mathrm{Er}^{3+}$ inverse opals for optical storage application. Chem. Eng. J. 394, 124967 (2020).

23. Ruan, J. F. et al. Thermomchromic reaction-induced reversible upconversion emission modulation for switching devices and tunable upconversion emission based on defect engineering of $\mathrm{WO}_{3}: \mathrm{Yb}^{3+}, \mathrm{Er}^{3+}$ phosphor. ACS Appl. Mater. Interfaces 10, 14941-14947 (2018).

24. Xu, W., Chen, X. \& Song, H. W. Upconversion manipulation by local electromagnetic field. Nano Today 17, 54-78 (2017).

25. Ohko, Y. et al. Multicolour photochromism of $\mathrm{TiO}_{2}$ films loaded with silver nanoparticles. Nat. Mater. 2, 29-31 (2003).

26. Ren, Y. T. et al. Reversible upconversion luminescence modification based on photochromism in $\mathrm{BaMgSiO}_{4} \mathrm{Yb}^{3+}, \mathrm{Tb}^{3+}$ ceramics for anti-counterfeiting applications. Adv. Optical Mater. 7, 1900213 (2019).

27. Grotjohann, T. et al. Diffraction-unlimited all-optical imaging and writing with a photochromic GFP. Nature 478, 204-208 (2011).

28. Zhang, C. et al. Luminescence modulation of ordered upconversion nanopatterns by a photochromic diarylethene: rewritable optical storage with nondestructive readout. Adv. Mater. 22, 633-637 (2010).

29. Zijlstra, P., Chon, J. W. M. \& Gu, M. Five-dimensional optical recording mediated by surface plasmons in gold nanorods. Nature 459, 410-413 (2009).

30. Wang, S. F. et al. Advances on tungsten oxide based photochromic materials: strategies to improve their photochromic properties. J. Mater. Chem. C 6, 191-212 (2018).

31. Dousti, M. R. et al. Structural and spectroscopic characteristics of Eu ${ }^{3+}$-doped tungsten phosphate glasses. Optical Mater. 45, 185-190 (2015).

32. Rabouw, F. T. et al. Non-blinking single-photon emitters in silica. Sci. Rep. 6, 21187 (2016).

33. Tarpani, $\mathrm{L}$. et al. Photoactivation of luminescent centers in single $\mathrm{SiO}_{2}$ nanoparticles. Nano Lett. 16, 4312-4316 (2016).

34. Schirmer, O. F. \& Salje, E. Conduction bipolarons in low-temperature crystalline WO $_{3-x .}$ J. Phys. C: Solid State Phys. 13, L1067 (1980).

35. Chen, M. Y. et al. Configurable phonon polaritons in twisted $\mathrm{a}-\mathrm{MoO}_{3}$. Nat. Mater. 19, 1307-1311 (2020).

36. Chan, J. W. et al. Fluorescence spectroscopy of color centers generated in phosphate glasses after exposure to femtosecond laser pulses. J. Am. Ceram. Soc. 85, 1037-1040 (2002).

37. Groot-Berning, K et al. Deterministic single-ion implantation of rare-earth ions for nanometer-resolution color-center generation. Phys. Rev. Lett. 123, 106802 (2019).

38. Baum, F. et al. Uncovering the mechanism for the formation of copper thioantimonate $\left(\mathrm{Sb}^{\mathrm{V}}\right)$ nanoparticles and its transition to thioantimonide ( $\left.\mathrm{Sb}^{\mathrm{II}}\right)$. Cryst. Growth Des. 18, 6521-6527 (2018).

39. Ashok, J. et al. Laser-stimulated piezo-optical and third harmonic generation studies for $\mathrm{Na}_{2} \mathrm{O}-\mathrm{Sb}_{2} \mathrm{O}_{3}$ glass ceramics-influence of gold ions. J. Mater. Sci.. Mater. Electron. 30, 3782-3791 (2019).

40. Noculak, A. et al. Bright blue and green luminescence of Sb(III) in double perovskite $\mathrm{Cs}_{2} \mathrm{MlnCl}_{6}(\mathrm{M}=\mathrm{Na}, \mathrm{K})$ matrices. Chem. Mater. 32, 5118-5124 (2020).

41. Eichelbaum, M. \& Rademann, K. Plasmonic enhancement or energy transfer? On the luminescence of gold-, silver-, and lanthanide-doped silicate glasses and its potential for light-emitting devices. Adv. Funct. Mater. 19, 2045-2052 (2009).

42. Zhou, B. et al. NIR II-responsive photon upconversion through energy migration in an ytterbium sublattice. Nat. Photonics 14, 760-766 (2020). 